\title{
VALIDAÇÃO DE METODOLOGIA ANALÍTICA POR CROMATOGRAFIA LÍQUIDA DE ALTA EFICIÊNCIA PARA QUANTIFICAÇÃO DE BUPIVACAÍNA (S75-R25) EM NANOESFERAS DE POLI(LACTÍDEO-CO- GLICOLÍDEO)
}

\author{
Carolina Morales Moraes e Eneida de Paula \\ Departamento de Bioquímica, Universidade Estadual de Campinas, Cidade Universitária “Zeferino Vaz”, 13083-970 \\ Campinas - SP, Brasil \\ André Henrique Rosa e Leonardo Fernandes Fraceto* \\ Departamento de Engenharia Ambiental, Universidade Estadual Paulista Júlio de Mesquita Filho, Av. Três de Março, 511, \\ 18087-180 Sorocaba - SP, Brasil
}

Recebido em 29/10/07; aceito em 14/5/08; publicado na web em 3/10/08

\begin{abstract}
VALIDATION OF ANALYTICAL METHODOLOGY BY HPLC FOR QUANTIFICATION OF BUPIVACAINE (S75-R25) IN POLI-LACTIDE-CO-GLICOLIDE NANOSPHERES. Bupivacaine (S75-R25, NovaBupi ${ }^{\circledR}$ ) is an amide type local anesthetic widely used. The present work consists of the development and validation of analytical methodology for evaluation of NovaBupi ${ }^{\circledR}$ content $^{2}$ in the poly-lactide-co-glycolide nanospheres (PLGA-NS) by high performance liquid chromatography. The separation was made using the reversed-phase column LC-18, acetonitrile/phosphate buffer 85:15 v/v as mobile phase and detection at $220 \mathrm{~nm}$. The results obtained show that the analytical methodology is accurate, reproducible, robust and linear over the concentration range 10-220.0 $\mu \mathrm{g} /$ $\mathrm{mL}$ of NovaBupi ${ }^{\circledast}$. The method was applied to determine the encapsulation efficiency and evaluate the release profile of NovaBupi ${ }^{\circledR}$, showing good results.
\end{abstract}

Keywords: bupivacaine; PLGA nanospheres; HPLC.

\section{INTRODUÇÃO}

A bupivacaína (BVC) é um anestésico local da classe das aminoamidas, que tem sido um dos fármacos de escolha na terapia da dor aguda e crônica. ${ }^{1,2}$ Seu uso na clínica se deve à potência e capacidade de produzir anestesia prolongada. A longa duração de ação, aliada à tendência em fornecer bloqueio mais sensorial que motor, tornou-a de uso popular para promover anestesia prolongada durante o trabalho de parto ou no período pós-operatório. ${ }^{3-5}$ Porém, sua supremacia foi questionada devido a relatos de cardiotoxicidade. ${ }^{6-8}$

Clinicamente, a cardiotoxicidade da BVC manifesta-se por arritmias ventriculares graves e depressão miocárdica após administração intravascular inadvertida de grandes doses. É provável que este efeito seja devido a vários fatores: a BVC bloqueia os canais de sódio cardíacos rapidamente durante a sístole, entretanto, a BVC se dissocia muito lentamente durante a diástole, de forma que uma fração significativa de canais de sódio permanece bloqueada com BVC no final da diástole. Portanto, o bloqueio com BVC é cumulativo e, substancialmente, maior do que seria previsível por sua potência anestésica local. ${ }^{1,9}$

A BVC, por possuir estereocentro, apresenta dois isômeros, dextrobupivacaína $(\mathrm{R}(+) \mathrm{BVC})$ e levobupivacaína (S(-)BVC), com comportamentos farmacológicos independentes em decorrência da estereosseletividade. ${ }^{10-12}$ Estudos demonstraram que a R(+)BVC é responsável pela cardiotoxicidade da BVC racêmica e pela sua refratariedade à reanimação cardiorrespiratória; a partir daí, desenvolveu-se a S(-)BVC, que comparada à BVC racêmica, é menos cardiotóxica e ligeiramente menos potente. . $^{2,6,13}$

Pesquisas sobre estereosseletividade levaram à modificação das proporções dos estereoisômeros $\mathrm{R}(+)$ e $\mathrm{S}(-)$ da $\mathrm{BVC}$ e à síntese de nova formulação anestésica local, contendo $25 \%$ do isômero $\mathrm{R}(+)$ e

*e-mail: leonardo@sorocaba.unesp.br
$75 \%$ do isômero $\mathrm{S}(-)$ da bupivacaína (denominada comercialmente de NovaBupi $\left.{ }^{\circledR}\right)$, melhorando o perfil anestésico do fármaco em relação à S(-)BVC e aumentando a margem de segurança, em relação à $\mathrm{BVC}$ racêmica. ${ }^{2,14}$

Como as características desejáveis para um anestésico local incluem longa duração de ação, seletividade para o bloqueio sensorial em relação ao motor e diminuição da toxicidade sistêmica, ${ }^{15,16}$ uma alternativa que tem se mostrado capaz de promover estes efeitos desejáveis é a liberação modificada destes fármacos. Esta veiculação de anestésicos locais pode ser obtida pela formação de complexos de inclusão com ciclodextrinas, ${ }^{2,9,12,14,17}$ encapsulação com lipossomas, ${ }^{18-22}$ associação em micropartículas ${ }^{22,23}$ e associação em nanopartículas poliméricas. ${ }^{24-27}$

Neste estudo, utilizando esta nova abordagem nanotecnológica de sistemas de liberação modificada para anestésicos locais, desenvolveram-se nanoesferas usando o polímero biodegradável poli(lactídeo-co-glicolídeo) (PLGA) como sistema de liberação para a NovaBupi ${ }^{\circledR}$. Este trabalho teve como objetivo desenvolver e validar uma metodologia analítica de quantificação confiável da NovaBupi ${ }^{\circledR}$ por cromatografia líquida de alta eficiência (CLAE), aplicar esta metodologia validada em breve estudo de quantificação da NovaBupi ${ }^{\circledR}$ na fase aquosa e acompanhar o sistema de liberação deste fármaco em sistemas contendo nanoesferas de PLGA.

\section{PARTE EXPERIMENTAL}

\section{Materiais}

O cloridrato bupivacaína (S75-R25) (NovaBupi ${ }^{\circledR}$ ) foi obtido por doação da Cristália Indústria Química e Farmacêutica Ltda. Os solventes empregados foram acetonitrila grau HPLC $\left(\right.$ Merck $\left.^{\circledR}\right)$ e água Milli-Q. Para preparo das nanoesferas foram utilizados poli(lactídeoco-glicolídeo) (PLGA) (50:50) de massa molecular 45000 g/mol 
$\left(\right.$ Sigma $\left.{ }^{\circledR}\right)$ e álcool polivinílico (PVA) $\left(\right.$ Sigma $\left.^{\circledR}\right)$. Os demais reagentes utilizados para preparo da solução tampão (fosfato de sódio monobásico e fosfato de sódio dibásico) e das nanoesferas (acetona e etanol) apresentavam grau espectroscópico analítico.

\section{Equipamentos}

Utilizou-se um cromatógrafo a líquido Shimadzu LC - 10A, bombas A e B (Shimadzu LC - 6AD), degaseificador (DGU-20AS), detector UV-VIS (SPD - 20AV), coluna cromatográfica Phenomenex, Gemini, $\mathrm{C}_{18}$ fase reversa com $5 \mu \mathrm{m}$ de tamanho de partícula, $150 \mathrm{~mm}$ de dimensão e 4,60 mm de diâmetro.

\section{Metodologia}

\section{Parâmetros cromatográficos}

A fase móvel utilizada foi constituída de acetonitrila e tampão fosfato $\mathrm{pH} 7,4,5 \mathrm{mM}(85: 15 \mathrm{v} / \mathrm{v})$ em fluxo de $1,2 \mathrm{~mL} / \mathrm{min}$ e a NovaBupi $^{\circledR}$ foi detectada pelo detector de ultravioleta (UV) em 220 nm. O volume de NovaBupi ${ }^{\circledast}$ injetado foi de $20 \mu \mathrm{L}$, sendo que todas as amostras injetadas, bem como a fase móvel, foram previamente filtradas em membrana de PTFE de 0,22 $\mu$ m (Millipore $^{\circledR}$ ).

O comprimento de onda foi escolhido a partir do espectro de absorção da BVC livre, onde se verificou que em $220 \mathrm{~nm}$ se tem máxima absorção. Injetou-se uma formulação placebo de NE, ou seja, NE composta por todos os componentes da formulação exceto o fármaco, e observou-se que no comprimento de onda escolhido os demais componentes da formulação não interferem no pico de absorção da BVC sendo, portanto, um comprimento de onda seletivo para BVC nesta situação.

\section{Validação da metodologia analítica}

A validação da metodologia analítica foi realizada segundo parâmetros estabelecidos pela Agência Nacional de Vigilância Sanitária (ANVISA) ${ }^{28}$ e International Conference on Harmonization of Technical Requirements for Registration of Pharmaceuticals for Human Use (ICH). ${ }^{29}$ Avaliou-se linearidade, precisão, exatidão, limite de quantificação e limite de detecção.

A linearidade do método foi determinada a partir de curvas analíticas obtidas em dois dias consecutivos $(n=6)$, apresentando cada uma 5 concentrações compreendidas entre 10 e $220 \mu \mathrm{g} / \mathrm{mL}$ de NovaBupi ${ }^{\circledR}$ diluída em acetonitrila.

As estimativas do limite de detecção (LD) e quantificação (LQ) foram realizadas a partir de dados obtidos com as regressões lineares das curvas analíticas aplicados às Equações 1 e 2, respectivamente.

$L D=\frac{D P \times 3}{I C}$

$L Q=\frac{D P \times 10}{I C}$

onde, DP é o desvio padrão do intercepto com o eixo y e IC, a inclinação da curva analítica média.

A repetibilidade ou precisão foi avaliada a partir do cálculo de desvio padrão relativo (DPR) (Equação 3).

$D P R=\frac{D P}{C M D} \times 100$

onde, DP é o desvio padrão e CMD, a concentração média determinada.

A exatidão foi determinada através de valores de concentração determinados experimentalmente, comparados à concentração teórica (Equação 4).
Exatidão $=\frac{C M D}{C T} \times 100$

onde, CT é a concentração teórica.

Para determinação da precisão e exatidão foram injetadas 3 amostras de NovaBupi ${ }^{\circledR}$ nas concentrações de 30, 60 e $120 \mu \mathrm{g} / \mathrm{mL}$ em triplicata em dois dias consecutivos.

\section{Preparo das nanoesferas de PLGA com NovaBupi ${ }^{\circledR}$}

A preparação das nanoesferas de PLGA foi realizada segundo o método de nanoprecipitação, envolvendo a mistura de uma fase orgânica em outra aquosa. A fase orgânica foi constituída por polímero PLGA, acetona e NovaBupi ${ }^{\circledR}$ (S75-R25). A fase aquosa foi composta por um tensoativo, o PVA e tampão fosfato $\mathrm{pH} 7,4$. Após dissolução dos componentes de ambas as fases, a fase orgânica foi injetada, lentamente, sob a fase aquosa com o auxílio de um funil. A suspensão resultante foi mantida sob agitação e, em seguida, foi concentrada até o volume de $10 \mathrm{~mL}$, com auxílio de um evaporador rotativo. ${ }^{30}$

\section{Quantificação de NovaBupi ${ }^{\circledR}$ nas nanoesferas}

O total $(100 \%)$ de NovaBupi ${ }^{\circledR}$ presente na suspensão de nanoesferas de PLGA foi determinado diluindo-se a suspensão em acetonitrila. A acetonitrila desestabiliza o sistema, liberando totalmente o fármaco, que foi quantificado a partir da equação da reta da curva analítica validada. A porcentagem de NovaBupi ${ }^{\circledR}$ associada às nanoesferas foi determinada pelo método de ultrafiltração/centrifugação, que consiste em submeter a suspensão de nanoesferas à centrifugação em dispositivos de ultrafiltração constituídos de celulose regenerada de $30 \mathrm{kDa}$ (Microcon - Millipore ${ }^{\circledR}$ ) e quantificar por CLAE o ultrafiltrado, utilizando-se a equação da reta obtida com a curva analítica validada. Só passa pela membrana de $30 \mathrm{kDa}$ o fármaco livre, sendo assim, relacionando-se a concentração total $(100 \%)$ de NovaBupi ${ }^{\circledR}$ presente na suspensão de nanoesferas e a concentração de NovaBu$\mathrm{pi}^{\circledR}$ não associada é possível determinar a porcentagem de fármaco associado nas nanopartículas. ${ }^{31-33}$ As medidas foram realizadas em três lotes, sendo analisadas triplicatas de cada lote.

\section{Teste de liberação in vitro}

Nos testes de liberação foram utilizados sistemas de dois compartimentos, separados por membranas de celulose (Spectrapore) de poro para exclusão molecular de $1000 \mathrm{Da}$, que permitiram observar a liberação da NovaBupi ${ }^{\circledR}(0,25 \%)$ em presença de nanoesferas de PLGA, desde o compartimento doador $(2 \mathrm{~mL})$ até o compartimento aceptor $(200 \mathrm{~mL})$, contendo tampão fosfato $50 \mathrm{mM}$, pH 7,4 sob agitação leve. Amostras do compartimento aceptor foram coletadas, durante $6 \mathrm{~h}$, e a concentração do anestésico foi determinada por CLAE nas condições descritas acima. O perfil de liberação foi observado através de gráficos de \% anestésico liberado em função do tempo. Os experimentos foram realizados em triplicata. A quantificação da porcentagem de AL liberada foi realizada utilizando as curvas de calibração validada descritas acima.

\section{RESULTADOS E DISCUSSÃO}

As condições cromatográficas utilizadas foram consideradas adequadas, pois foi obtido um pico simétrico da NovaBupi ${ }^{\circledR}$ (Figura 1) e viável, pois foram realizadas corridas cromatográficas de apenas 3 min. O método também se mostrou específico, uma vez que no cromatograma do placebo não se detectou absorção no tempo de retenção correspondente ao pico cromatográfico da bupivacaína (cromatograma não mostrado).

A linearidade do método foi avaliada a partir de regressões lineares de seis curvas analíticas (Figura 2). O coeficiente de correlação 


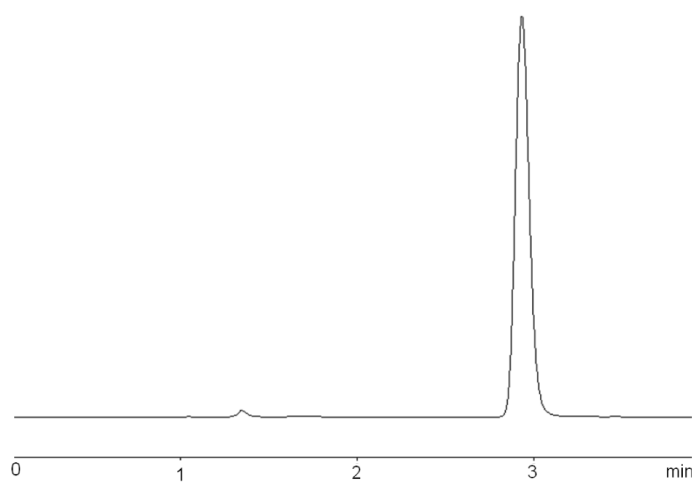

Figura 1. Cromatograma da NovaBupi ${ }^{\circledR}$

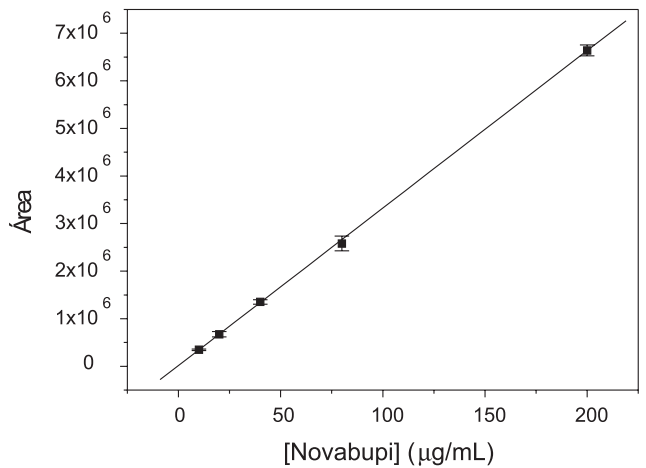

Figura 2. Curva analítica da NovaBupi $i^{\oplus}(n=6)$ e parâmetros obtidos com a regressão linear da curva analítica

(r) da curva analítica média ( $\mathrm{n}=6$ ) foi de 0,9999 e na Tabela 1 tem-se os coeficientes de correlação (r) das curvas de calibração realizadas a cada dia, durante dois dias consecutivos, sendo cada curva em triplicata. O coeficiente de correlação é um parâmetro que permite uma estimativa da qualidade da curva obtida, pois quanto mais próximo de 1,0 , menor a dispersão do conjunto de pontos experimentais. ${ }^{34} \mathrm{~A}$ ANVISA recomenda um coeficiente de correlação maior ou igual a 0,99 e todos os valores de correlação linear obtidos estão acima deste valor, sendo o método, portanto, considerado linear. ${ }^{28}$

A partir dos dados obtidos com as regressões lineares aplicadas às Equações 1 e 2, determinaram-se o limite de detecção $(0,54 \mu \mathrm{g} /$ $\mathrm{mL})$ e o limite de quantificação $(1,79 \mu \mathrm{g} / \mathrm{mL})$.

A repetibilidade ou precisão intra-dia foi determinada a partir da determinação do desvio padrão relativo (DPR) intra-dia e a precisão intermediária ou precisão inter-dias foi determinada a partir da determinação do DPR inter-dias. Estes valores de DPR estão expressos na Tabela 2.

A precisão é a avaliação da proximidade dos resultados obtidos de uma mesma amostra. Os valores de DPR determinados intra-dia e inter-dias foram todos abaixo do valor preconizado pela ANVISA, que é de no máximo $5 \%$, portanto o método pode ser considerado preciso. ${ }^{28}$

A exatidão foi determinada através de valores de concentração determinados experimentalmente, comparados à concentração teórica, como se pode observar na Tabela 3. Na Tabela 4 estão expressos os valores dos percentuais de recuperação do fármaco.

Tabela 1. Coeficientes de correlação (r) das curvas de calibração em dois dias consecutivos

\begin{tabular}{lcc}
\hline & Dia 1 $(n=3)$ & Dia 2 $(n=3)$ \\
\hline $\mathrm{r}$ & 0,9999 & 0,9992 \\
\hline
\end{tabular}

Tabela 2. Valores de desvio padrão relativo (DPR) das concentrações de NovaBupi ${ }^{\circledR}$ determinados para avaliação da precisão

\begin{tabular}{lccc}
\hline$[\mathrm{BVC}](\mu \mathrm{g} / \mathrm{mL})$ & DPR dia $1(\%)$ & DPR dia $2(\%)$ & DPR inter-dias $(\%)$ \\
\hline 30 & 1,126 & 3,563 & 3,627 \\
60 & 0,839 & 1,837 & 2,591 \\
120 & 1,455 & 0,819 & 1,993 \\
\hline
\end{tabular}

A exatidão é a relação entre a concentração média determinada experimentalmente e a concentração teórica correspondente. A porcentagem de recuperação variou de 99,9 a $104,5 \%$, estando dentro das especificações estabelecidas. ${ }^{28}$

A quantificação de BVC nas nanoesferas de PLGA foi determinada através da equação da reta da curva analítica validada (Figura 2). As concentrações de BVC presentes na suspensão de nanoesferas (100\%) e não associadas às nanoesferas estão na Tabela 5. A partir destes dados, calculou-se a porcentagem de BVC associada nas nanoesferas e o valor encontrado foi de 5,09 $\pm 0,01 \%$. O valor relativamente baixo de associação pode estar associado à alta concentração de BVC utilizada $(0,25 \mathrm{mg} / \mathrm{mL})$ no preparo da suspensão de nanopartículas e, assim, a parte que está associada às nanoesferas, saturando-as, se torna pouca se comparada ao todo, entretanto, tem-se uma concentração de $0,0125 \mathrm{mg} / \mathrm{mL}$ de BVC associada às nanopartículas. Esta concentração de moléculas de BVC incorporadas às nanoesferas de PLGA apresentou diminuição na citotoxicidade celular em culturas de células de fibroblastos (dados não mostrados). Além disso, a razoável solubilidade deste fármaco em água pode estar levando à perda de BVC para a água, diminuindo a associação às nanoesferas.

Trabalhos da literatura ${ }^{24,26}$ descrevem para a lidocaína (um anestésico local) uma taxa associação entre 6 e $30 \%$ em nanoesferas de PLA, destacando que a taxa de associação é dependente da forma do anestésico, no caso na forma base livre, com menor solubilidade em

Tabela 3. Valores de concentração de NovaBupi ${ }^{\circledR}$ determinados para avaliação da exatidão

\begin{tabular}{lccc}
\hline $\begin{array}{l}{\left[\mathrm{BVC}^{*}\right]} \\
(\mu \mathrm{g} / \mathrm{mL})\end{array}$ & $\begin{array}{c}{[\mathrm{BVC} * *] \pm \mathrm{DP}} \\
(\mu \mathrm{g} / \mathrm{mL}) \text { dia } 1\end{array}$ & $\begin{array}{c}{[\mathrm{BVC} * *] \pm \mathrm{DP}} \\
(\mu \mathrm{g} / \mathrm{mL}) \text { dia } 2\end{array}$ & $\begin{array}{c}{\left[\mathrm{BVC}^{* *}\right] \pm \mathrm{DP}} \\
(\mu \mathrm{g} / \mathrm{mL}) \text { inter-dias }\end{array}$ \\
\hline 30 & $29,9 \pm 0,1$ & $31,0 \pm 0,1$ & $30,4 \pm 0,1$ \\
60 & $62,7 \pm 0,3$ & $60,2 \pm 0,1$ & $61,4 \pm 0,2$ \\
120 & $124,7 \pm 0,1$ & $120,9 \pm 0,2$ & $122,8 \pm 0,2$ \\
\hline
\end{tabular}

* concentração de bupivacaina teórica; ** concentração de bupivacaína experimental

Tabela 4. Valores de porcentagem de recuperação de NovaBupi ${ }^{\circledR}$ determinados para avaliação da exatidão

\begin{tabular}{lccc}
\hline $\begin{array}{l}\text { BVC*] } \\
(\mu \mathrm{g} / \mathrm{mL})\end{array}$ & $\begin{array}{c}\text { \% de recuperação } \\
\text { dia } 1\end{array}$ & $\begin{array}{c}\text { de recuperação \% de recuperação } \\
\text { dia } 2\end{array}$ & $\begin{array}{c}\text { inter-dias } \\
\text { ind }\end{array}$ \\
\hline 30 & 99,9 & 104,9 & 102,4 \\
60 & 104,5 & 100,3 & 102,4 \\
120 & 103,9 & 100,8 & 100,3 \\
\hline
\end{tabular}

* concentração de bupivacaína teórica

Tabela 5. Valores médios de concentração de NovaBupi ${ }^{\circledR}$ determinados em estudos de eficiência de associação em nanoesferas

\begin{tabular}{lcc}
\hline $\begin{array}{l}{[\mathrm{BVC}] \text { total }(100 \%)} \\
(\mathrm{mg} / \mathrm{mL}) \pm \mathrm{DP}(\mathrm{n}=9)\end{array}$ & $\begin{array}{c}{[\mathrm{BVC}] \text { não associada }} \\
\text { às nanoesferas } \\
(\mathrm{mg} / \mathrm{mL}) \pm \mathrm{DP}(\mathrm{n}=9)\end{array}$ & $\%$ de associação \\
\hline $2,25 \pm 0,02$ & $2,14 \pm 0,01$ & $5,09 \pm 0,01$ \\
\hline
\end{tabular}


água (comparada à forma ionizada) e, também, que um aumento na proporção de polímero faz com que os tamanhos das esferas produzidas sejam aumentadas e, por conseqüência, a taxa de associação.

A fim de verificar a eficiência da curva analítica validada, foi realizado um ensaio de liberação de NovaBupi ${ }^{\circledR}$ contendo nanoesferas de PLGA, durante um período de 6 h (360 min, Figura 3). Neste ensaio, demonstra-se que neste período a totalidade de NovaBupi ${ }^{\circledR}$ foi liberada, sendo a curva analítica útil para quantificação do fármaco liberado em função do tempo.

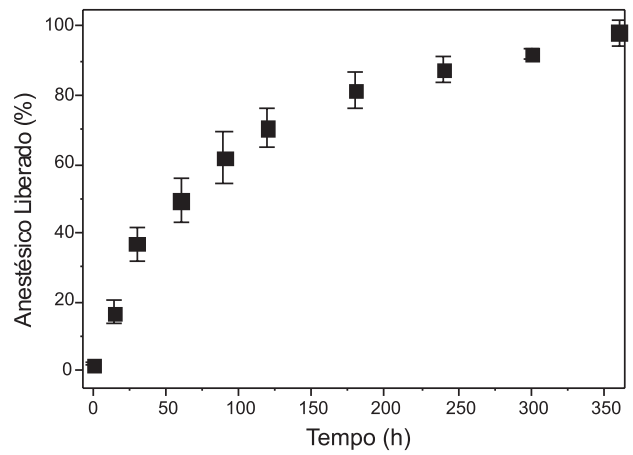

Figura 3. Teste de liberação in vitro para NovaBupi $i^{\circledR}$ em presença de nanoesferas de PLGA, $p H$ 7,4, temperatura ambiente, em função do tempo $(n=3)$

\section{CONCLUSÃO}

A metodologia analítica de quantificação da NovaBupi ${ }^{\circledast}$, validada segundo ANVISA (2003) e ICH (1996), foi considerada rápida e viável, uma vez que cada corrida cromatográfica durou apenas $3 \mathrm{~min}$, além de apresentar linearidade na faixa de concentração proposta, exatidão e precisão, atendendo às exigências analíticas e assegurando a confiabilidade dos resultados. Foram determinados também os limites de quantificação e de detecção, indicando que a metodologia é eficiente para quantificar BVC em concentração mínima de 1,79 $\mu \mathrm{g} / \mathrm{mL}$. Além disso, o limite de quantificação torna a metodologia adequada à utilização em ensaios de liberação.

O método validado mostrou-se adequado para a quantificação de NovaBupi ${ }^{\circledR}$ na fase aquosa em preparações contendo nanoesferas de PLGA em ensaios que determinam a porcentagem de associação de fármaco em nanopartículas, avaliação fundamental para o desenvolvimento desta nova formulação de suspensão de nanoesferas proposta.

\section{AGRADECIMENTOS}

À FAPESP pelo auxílio financeiro (05/03045-9, 06-00121-9 e 07/00127-0) e bolsa de mestrado (C. M. Moraes, 06/05220-5). A. H. Rosa e E. de Paula agradecem ao CNPq pela bolsa de produtividade em pesquisa. Os autores agradecem também as sugestões e contribuições dos assessores da revista.

\section{REFERÊNCIAS}

1. Gupta, S. P.; Chem. Rev. 1991, 91, 1109.

2. de Araújo, D. R.; Fraceto, L. F.; Braga, A. F. A.; de Paula, E.; Rev. Bras. Anestesiol. 2005, 55, 316.

3. Goodman, A. G.; Gilman, A. G.; As Bases Farmacológicas da Terapêutica, McGraw-Hill: Rio de Janeiro, 1996.

4. Mclure, H. A.; Rubin, A. P.; Minerva Anestesiol. 2005, 71, 59.
5. David, J. S.; Ferreti, C.; Amour, J.; Vivien, B.; Eve, O.; Petit, P.; Riou, B.; Gueugniaud, P. Y.; Can. J. Anesth. 2007, 54, 208.

6. Albright, G. A.; Anesthesiology 1979, 51, 285.

7. Malamed, S. F.; Manual de anestesia local, Guanabara Koogan: Rio de Janeiro, 2001.

8. Udelsmann, A.; Lorena, S. E. R. S.; Girioli, S. U.; Silva, W. A.; Moraes, A. C.; Rev. Bras. Anestesiol. 2007, 57, 63.

9. Moraes, C. M.; Abrami, P.; de Paula, E.; Andreo Filho, N.; Fraceto, L. F.; Quim. Nova 2007, 30, 777; Moraes, C. M.; Abrami, P.; de Araújo, D. R.; Braga, A. F. A.; Issa, M. G.; Ferraz, H. G.; de Paula, E.; Fraceto, L. F.; J. Incl. Phenom. Macrocycl. Chem. 2007, 57, 313.

10. Clarkson, C. W.; Hondeghem, L. M.; Anesthesiology 1985, 62, 396.

11. Casati, A.; Santorsola, R.; Aldegheri, G.; Ravasi, F.; Fanelli, G.; Berti, M.; Franchini, G.; Torri, G.; J. Clin. Anesth. 2003, 15, 126.

12. Moraes, C. M.; Abrami, P.; de Paula, E.; Braga, A. F.; Fraceto, L. F.; Int. J. Pharm. 2007, 331, 99.

13. Cangiani, L. H.; CangianI, L. M.; Pereira, A. M. S. A.; Rev. Bras. Anestesiol. 2007, 57, 136.

14. de Araújo, D. R.; Braga, A. F. A.; Moraes, C. M.; Fraceto, L. F.; de Paula, E.; Rev. Bras. Anestesiol. 2006, 56, 495.

15. Kuzma, P. J.; Kline, M. D.; Calkins, M. D.; Staats, P. S.; Reg. Anesth. 1997, 22, 543

16. de Araújo, D. R.; Pinto, L. M. A.; Braga, A. F. A.; de Paula, E.; Rev. Bras. Anestesiol. 2003, 53, 663.

17. Pinto, L. M.; Fraceto, L. F.; Santana, M. H. A.; Pertinhez, T. A.; Junior, S. O.; de Paula, E.; J. Pharm. Biomed. Anal. 2005, 39, 956.

18. Grant, G. J.; Bansinath, M.; Reg. Anesth. Pain Med. 2001, $26,61$.

19. Grant, S. A.; Best Pract. Res. Clin. Anaesth. 2002, 16, 345.

20. de Araújo, D. R.; Cereda, C. M. S.; Brunetto, G. B.; Pinto, L. M. A.; Santana, M. H. A.; de Paula, E.; Can. J. Anesth. 2004, 51, 566.

21. Cereda, C. M. S.; de Araújo, D. R.; Brunetto, G. B.; de Paula, E.; J. Pharm. Pharmacol. Sci. 2004, 7, 235.

22. Rose, J. S.; Neal, J. M.; Kopacz, D. J.; Reg. Anesth. Pain Med. 2005, 30, 275.

23. Le Guévello, P.; Le Corre, P.; Chevanne, F.; Le Verge, R.; J. Chromatogr. 1993, 622, 284.

24. Gorner, T.; Gref, R.; Michenot, D.; Sommer, F.; Tran, M. N.; Dellacherie, E.; J. Controlled Release 1999, 57, 259.

25. Govender, T.; Stolnik, S.; Garnett, M. C.; Illum, L.; Davis, S. S.; J. Controlled Release 1999, 57, 171.

26. Polakovic, M.; Gorner, T.; Gref, R.; Dellacherie, E.; J. Controlled Release 1999, 60, 169.

27. Govender, T.; Riley, T.; Ehtezazi, T.; Garnett, M. C.; Stolnik, S.; Illum, L.; Davis, S. S.; Int. J. Pharm. 2000, 199, 95.

28. Agência Nacional de Vigilância Sanitária, resolução RE nº 899, de 23 de maio de 2003, Brasil.

29. Guidance for Industry - Q2B Validation of Analytical Procedures: Methodology, European Agency for the Evaluation of Medicinal Products (ICH): Londres, 1996

30. Fessi, H.; Puiseiux, F.; Devissaguet, J-P. ; Procèdé de preparation de systèmes colloidaux displesibles d'une substance sous forme de nanocapsules, Eur. Pat., 0274961 A1, 1988.

31. Gamisans, F.; Lacoulonche, F.; Chauvet, A.; Espina, M.; Garcia, M. L.; Egea, M. A.; Int. J. Pharm. 1999, 179, 37.

32. Schaffazick, S. R.; Guterres, S. S.; Freitas, L. L.; Pohlmann, A. R.; Quim. Nova 2003, 26, 726.

33. Kilic, A. C.; Capan, Y.; Vural, I.; Gursoy, R. N.; Dalkara, T.; Cuine, A.; Hincal, A. A.; J. Microencapsulation 2005, 22, 633.

34. Ribani, M.; Bottoli, C. B. G.; Collins, H. C.; Jardim, I. C. S. F.; Melo, L. F. C.; Quim. Nova 2004, 27, 771. 